The Future Astronuclear Physics

H.M.J. Boffin, S. Goriely, A. Jorissen, \& $M$. Rayet

EAS Publications Series, Vol. ?, 2004

\title{
CORE-COLLAPSE SUPERNOVAE INDUCED BY ANISOTROPIC NEUTRINO RADIATION
}

\author{
Yuko Motizuki $^{1}$, Hideki Madokoro ${ }^{1}$ and Tetsuya Shimizu ${ }^{1}$
}

\begin{abstract}
We demonstrate the important role of anisotropic neutrino radiation on the mechanism of core-collapse supernova explosions. Through a new parameter study with a fixed radiation field of neutrinos, we show that prolate explosions caused by globally anisotropic neutrino radiation is the most effective mechanism of increasing the explosion energy when the total neutrino luminosity is given. This is suggestive of the fact that the expanding materials of SN 1987A has a prolate geometry.
\end{abstract}

\section{Introduction}

In spite of every effort over more than 30 years since the first work of Colgate \& White (1966), the mechanism of core-collapse supernovae is still under debate. In the so-called "delayed explosion" scenario, which is believed to be responsible in the mechanism of core-collapse supernovae, the supernova shock is once stagnated around $\sim 150-200 \mathrm{~km}$ above the neutrinosphere. Then neutrinos heat the matters behind the stalled shock, and finally help the shock wave to revive or move upward, resulting in an explosion. So far, no "successful" supernova simulations have been reported except work by Wilson and Mayle $(1988,1993)$. Here a "successful" supernova explosion must explain the following observed facts: 1) the explosion energy (observed in SN 1987A): $1.5 \pm 0.5 \times 10^{51}$ ergs, 2) asymmetry observed by spectropolarimetry measurements in SN 1987A, SN 1993J, and other several supernovae, 3 ) remnant neutron star mass of $1.4 M_{\odot}$, and 4) amount of explosive nucleosynthesis of ${ }^{56} \mathrm{Ni}: \sim 0.07 M_{\odot}$ in the case of SN 1987A.

It has been theoretically demonstrated that simulations assuming spherical symmetry fail to produce robust explosions even if general relativistic Boltzmann neutrino transport is taken into account (Liebendörfer et al. 2001). Therefore together with the above-mentioned observed asymmetries in the explosions 2), we call for multi-dimensional hydrodynamical simulations. At this time, such simulations have been performed for two-dimensional (2D) models (Miller et al. 1993;

${ }^{1}$ RIKEN, Hirosawa 2-1, Wako 351-0198 Japan 
The Future Astronuclear Physics

Herant et al. 1994; Burrows et al. 1995; Janka \& Müller 1996; Mezzacappa et al. 1998; Fryer \& Heger 2000; Shimizu et al. 2001; Buras et al. 2003) and threedimensional models (Shimizu et al. 1993; Fryer \& Warren 2002). In these simulations, special attention is paid to the role of convection either near the surface of a nascent neutron star or in neutrino-heated regions above the neutrinosphere. It has been argued that large-scale mixing, caused by convection and convective overturn around the neutrino-heated region, increases the average entropy and therefore the explosion energy, and this can trigger a successful explosion (e.g., Herant et al. 1994; Janka \& Müller 1996). It is noted, however, that almost all hydrodynamical simulations so far have been performed with spherically symmetric neutrino radiation field. In this article, we show that anisotropic neutrino radiation, or locally intense neutrino heating, can be an alternative mechanism to revive the shock wave and to lead to a successful explosion other than the so far suggested "convective trigger".

Shimizu and coworkers (Shimizu et al. 1994; Shimizu et al. 2001) first proposed that the anisotropic neutrino radiation should play a crucial role in the explosion mechanism and carefully investigated the effects of anisotropic neutrino radiation on the explosion energy. They performed 2D hydrodynamical simulations and found that only a few percent of enhancement in the neutrino flux along the axis of symmetry is sufficient to increase the explosion energy by a large factor, and that this effect saturates around a certain degree of anisotropy.

The origin of anisotropic neutrino radiation is considered as follows. Because supernova progenitors such as OB stars are generally observed to be fast rotators ( $\sim 200 \mathrm{~km} \mathrm{~s}^{-1}$ at the surface, $P \sim 1$ day; see, e.g., Fukuda 1982), the resulting proto-neutron stars can have a large amount of angular momentum after the gravitational collapse. Centrifugal force then deforms the rotating core into an oblate form. This will cause asymmetric neutrino radiation, in which the flux along the pole is enhanced over that on the equatorial plane. Anisotropy in neutrino radiation may also be originated from the convection inside the proto-neutron star, or asymmetric mass accretion onto the neutron star. Recently, Kotake et al. (2003) performed 2D simulations of the rotational collapse of a supernova core and found that the rotation can actually produce stronger neutrino radiation in the direction of the rotational axis than that on the equator.

Neutrino flux can also fluctuate with angle and time as suggested by Burrows et al. (1995), due to gravitational oscillation of the neutrinosphere. In this article we carry out 2D hydrodynamical simulations with a fixed radiation field of neutrinos, to investigate what kind of neutrino radiation is favorable for a successful explosion. We briefly demonstrate that globally anisotropic (prolate) neutrino radiation is the most effective way of increasing the explosion energy compared with spherically symmetric and fluctuated neutrino radiation. Details are found in Madokoro et al. (2003, 2004). 


\section{General Features of an Explosion Induced by Anisotropic $\nu$ Radiation}

In this section we describe our simplifications to the problem and general features of an explosion induced by anisotropic neutrino radiation. We perform $2 \mathrm{D}$ simulations by solving hydrodynamical equations in the spherical coordinate $(r, \theta)$. A generalized Roe's method is used to solve the general equations of motion. In order to set the initial condition, we use the fact that, in the delayed mechanism, the shock is stalled at a radius of a few hundred kilometers and stays for a few hundred milliseconds. We reproduce the stalled shock wave at the radius of $200 \mathrm{~km}$ by solving stationary hydrodynamic equations with assuming spherical symmetry, and start calculations when a stalled shock wave is formed. It is a good approximation to exploit the stationary hydrodynamic solution as an initial model. Here the radius of the proto-neutron star is fixed to be $50 \mathrm{~km}$ and the computational region ranges from $50 \mathrm{~km}$ to $10000 \mathrm{~km}$ in radius from the center of the proto-neutron star. The details of our numerical technique, the adopted equation of state, and the initial conditions are described in the previous papers (Shimizu et al. 2001; Madokoro et al. 2003). It is noted here that we have improved the numerical code in these papers to avoid a numerical error near the pole. Note that this numerical error was not serious and minor for the investigation of the explosion energy, but may affect the results of nucleosynthesis.

The local neutrino flux seen by an observer positioned at a distance $r$ far from the neutrinosphere and at the angle $\theta$ from the axis of symmetry is assumed as

$$
l_{\nu}(r, \theta)=\frac{7}{16} \sigma T_{\nu}^{4} c_{1}\left(1+c_{2} \cos ^{2} \theta\right) \frac{1}{r^{2}},
$$

where $\sigma$ is the Stefan-Boltzmann constant and $T_{\nu}$ is the neutrino temperature of assumed blackbody radiation. In our simulations $T_{\nu}$ is taken to be constant and hence the total neutrino luminosity, $L_{\nu}$, which is obtained by integrating Eq. (2.1) over the solid angle, is also constant in time. Recent calculations (e.g., Fryer \& Heger 2000) have suggested that the decay timescale of the neutrino luminosity is about $500 \mathrm{~ms}$, and hence we stop our calculations with constant $L_{\nu}$ at $\mathrm{t}=500 \mathrm{~ms}$ after the shock stagnation. The above simplifications are enough for our purpose (see Madokoro et al. 2003 for details).

In Eq. (2.1), the parameter $c_{2}$ represents the magnitude of anisotropy in the neutrino radiation. One can easily confirm that the neutrino fluxes in the polar and equatorial directions, $l_{z} \equiv l_{\nu}(r, \theta=0)$ and $l_{x} \equiv l_{\nu}(r, \theta=\pi / 2)$, are proportional to $c_{1}\left(1+c_{2}\right)$ and $c_{1}$, respectively. The degree of anisotropy seen from each respective observer is then given by

$$
\left[\frac{l_{z}}{l_{x}}\right]_{o b s}=1+c_{2} .
$$

This is schematically illustrated in Fig. 1. The value of $c_{1}$ in Eq. (2.1) is calculated from given $c_{2}$ so as to adjust the total neutrino luminosity $L_{\nu}$ to that in the spherical model at the same $T_{\nu}$.

Figure 2 depicts the dimensionless entropy contour and the velocity field to explain the general feature of an explosion induced by anisotropic neutrino radia- 
prolate $\left(\mathrm{c}_{2}>0\right)$

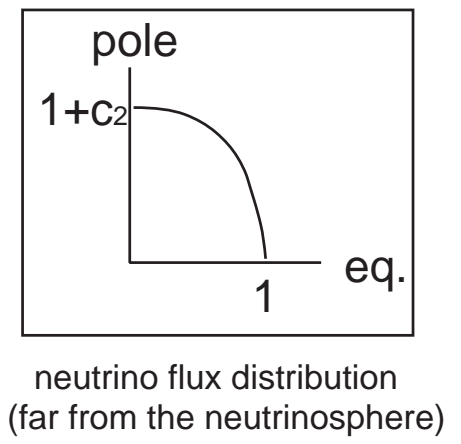

Fig. 1. A schematic picture that shows the neutrino flux distribution seen from each respective observer far from the neutrinosphere.

tion. As described above, a stronger neutrino radiation is assumed to be emitted in the direction of the symmetric (polar) axis of a proto-neutron star than in the equatorial direction. The matter surrounding the neutron star is first heated along the pole, and a high-entropy region is formed there. Since there is a gravitational pull by the central neutron star, the high-entropy bubble moves upward as a result of buoyancy. The upward velocity field can be clearly seen in Fig. 2. The high pressure of the heated matter then pushes up the shock front, which deviates from spherical symmetry. This asymmetric, jetlike explosion is a common feature as a consequence of anisotropic neutrino radiation. It has been found that the hot bubble evolves faster with increasing the anisotropy in the neutrino radiation field. The large mushroom-like structure of the hot bubble is produced, as seen in Fig. 2, as a result of the global circular motion behind the shock front. The circulation is global and clockwise in the figure, and its cycle is only a few rotations per explosion. The distortion of the shock wave continues and not diminished in the course of the shock propagation.

\section{Explosion Energy Increase Triggered by Anisotropic $\nu$ Radiation}

We are going to see that the explosion energy dramatically increases as the result of anisotropic neutrino radiation. Figure 3 shows the evolution of the explosion energy, as well as the thermal, kinetic, and gravitational energies. The thick solid line of the explosion energy $\left(E_{\text {expl }}\right)$ represents the explosion caused by anisotropic neutrino radiation for the model of $\left[l_{z} / l_{x}\right]_{o b s}=1.1$ and $T_{\nu}=4.65 \mathrm{MeV}$. The dotted line of $E_{\operatorname{expl}}$ in Fig. 3 is for the case when the neutrino field is fluctuated in space (Burrows et al. 1995). The thin solid line of $E_{\text {expl }}$ in Fig. 3, which is difficult to distinguish from the dotted line although, shows the case for the spherical explo- 
$\phi$ colorscale \& velocity field

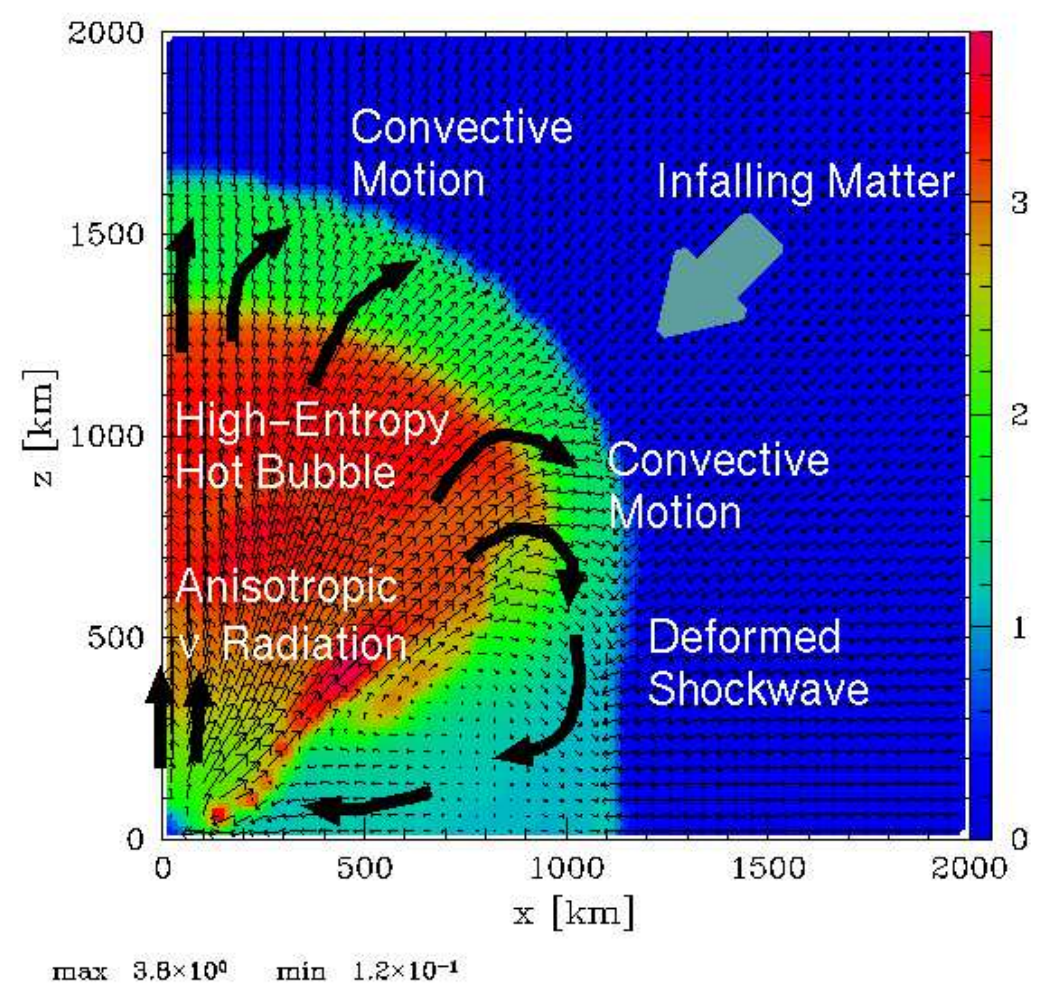

Fig. 2. Dimensionless entropy contour map with the velocity field for the model of $\left[l_{z} / l_{x}\right]_{o b s}=1.1$ and $T_{\nu}=4.7 \mathrm{MeV}$ at $\mathrm{t}=160 \mathrm{~ms}$ after the shock stall. Note that the shock wave itself is deformed.

sion. We see in Fig. 3 that the simulations fail to explode when the neutrino field is spherically symmetric and fluctuated, but may produce an adequate explosion when the field is reasonably anisotropic: The difference between the model with global anisotropy and the other models is extremely remarkable. The degree of anisotropy assumed here, $\left[l_{z} / l_{x}\right]_{o b s}=1.1$, can be translated into the rotational period of $\sim 15 \mathrm{~ms}$ on the assumption of the Maclaurin spheroid, and hence this is considered to be in the reasonable range as the rotational period of a proto-neutron star.

In the previous studies we have performed simulations with various types of models and concluded that the global anisotropy of the neutrino radiation is the most effective mechanism of increasing the explosion energy among spherically symmetric and fluctuated neutrino radiating models when the total neutrino luminosity is given. We also found that the effect of anisotropic neutrino radiation 


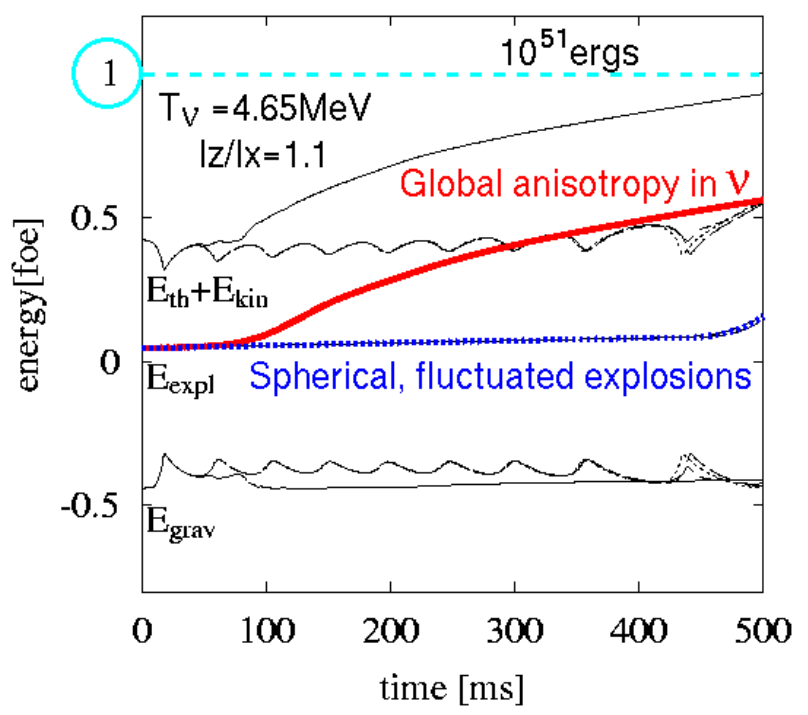

Fig. 3. Evolution of thermal and kinetic energy $\left(\mathrm{E}_{\mathrm{th}}+\mathrm{E}_{\mathrm{kin}}\right)$, gravitational energy $\left(\mathrm{E}_{\mathrm{grav}}\right)$, and explosion energy $\left(\mathrm{E}_{\text {expl }}\right)$ for the anisotropic model of $\left[l_{z} / l_{x}\right]_{o b s}=1.1$ and $T_{\nu}=4.65$ $\mathrm{MeV}$, and spherical and fluctuated neutrino radiating models (see the text).

on the explosion energy appears with a small value $\left(\left[l_{z} / l_{x}\right]_{\text {obs }} \sim 1.05\right)$ and the effect saturates with a certain value $\left(\left[l_{z} / l_{x}\right]_{o b s} \sim 1.2\right)$. We emphasize that a large anisotropy in the neutrino radiation is not required for the explosion mechanism. We also remark that Shimizu et al. (2001) reported that the anisotropy of $\left[l_{z} / l_{x}\right]_{\text {obs }}$ $=1.2$ in the neutrino radiation is equivalent to a convective model with an initial perturbation of $10 \%$ in the density of the infalling material, as far as the effect on the explosion energy is considered.

\section{Reasoning of Powerful Explosion Induced by Anisotropic $\nu$ Radiation}

We present in this section the reasoning of the powerful explosion caused by the globally anisotropic neutrino radiation. The crucial point here is that the balance between neutrino heating and cooling. This determines the evolution of the explosion energy. Here the neutrino heating is caused by neutrino absorption due to nuclei and neutrino scattering off electrons and positrons, and hence the heating rate depends on the neutrino temperature, $T_{\nu}$ : The heating rate varies as $T_{\nu}^{6}$. On the other hand, the neutrino cooling is caused by neutrino emission due to electron captures and also by thermal (photo, pair, plasma) neutrino emission processes. The cooling rate is then a steep function of the matter temperature $T$, roughly proportional to $T^{6}$.

Figure 4 shows the accumulation of the absorbed and emitted energies due to 


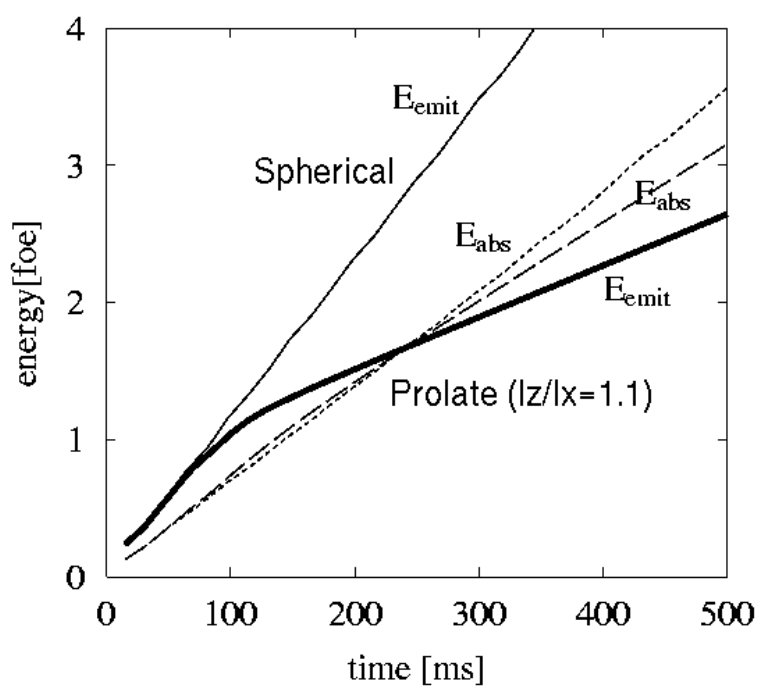

Fig. 4. Accumulation of the absorbed and emitted energies due to neutrino heating $\left(E_{\text {abs }}\right)$ and cooling $\left(E_{\text {emit }}\right)$. The thick solid line and the dashed line represent, respectively, $E_{\text {emit }}$ and $E_{\text {abs }}$ for the anisotropic model of $\left[l_{z} / l_{x}\right]_{o b s}=1.1$. The thin solid line and the dotted line represent $E_{\text {emit }}$ and $E_{\text {abs }}$, respectively, for the spherically symmetric model. For both models neutrino temperature $T_{\nu}=4.65 \mathrm{MeV}$ is adopted. Note the significant difference in the emitted energy.

neutrino heating and cooling for the globally anisotropic and for the spherical models. As mentioned before, the locally intense neutrino radiation in the anisotropic model results in local heating along the polar axis. The thermal pressure around the heated matter becomes high, and hence it pushes a part of the shock wave outward. Namely, the shock revival occurs in one direction first along the polar axis. Then the pressure gradient does work along the shock front, and the shock revival prevails into all directions (note the Rankine-Hugoniot condition). This leads to an earlier shock revival than the spherical model. As the shock expands, the matter temperature behind the shock decreases rapidly. The neutrino cooling rate, which is very sensitive to the matter temperature, accordingly drops rapidly. On the other hand, the neutrino heating rate remains almost unchanged between the the anisotropic and the spherical models. Heating dominates cooling as a result. These are seen in Fig. 4.

Eventually, the explosion energy increases because of the suppression of neutrino cooling. We note that the position where the cooling rate suddenly starts to decrease in Fig. 4 (i.e., $\mathrm{t} \sim 90 \mathrm{~ms}$ for the anisotropic model) is equivalent to the time when the explosion energy suddenly starts to rise up in Fig. 3. This time corresponds to the shock revival time. 
The Future Astronuclear Physics

\section{Concluding Remarks: Problems and the Future}

In this article it has been demonstrated that the explosion energy in the globally anisotropic neutrino radiation model increases most effectively among spherically symmetric and fluctuated radiating models when the total neutrino luminosity is given. As a matter of fact, the expanding materials of SN 1987A suggestively has a prolate geometry (Wang et al. 2002). We conclude that anisotropic neutrino radiation or locally intense neutrino heating can be alternative mechanism for a "successful" explosion other than the so far suggested convective trigger.

The enhancement of total neutrino luminosity itself is actually the most important ingredient in the delayed explosion mechanism for driving an energetic explosion as concluded by Janka \& Müller $(1995,1996)$. If the total neutrino luminosity adopted in the simulations is sufficiently large, any models may easily explode regardless of the type of the explosion. However, such treatment often leads to the problem of $\mathrm{Ni}$ overproduction, especially in the case of essentially spherical models. Because of this, the total neutrino luminosity cannot be simply increased. Our results show that the enhancement of the total neutrino luminosity is not always necessary. Self-consistent simulations of the rotating supernova core and anisotropic neutrino radiation from the beginning of the gravitational collapse are now under progress.

Since we do not know at present whether the neutrino luminosity in a real supernova explosion is strong enough or not, or whether it has been reproduced correctly in the past simulations, we have to wait for the answer to whether anisotropy in the neutrino radiation field is generally important or not, until the neutrino opacity and the numerical technique for the neutrino transport are improved in future calculations. Further studies on nuclear equation of state, inelastic neutrino scattering, and weak interactions on nuclei at subnuclear densities and at

finite temperatures are indeed required. Also, future detections of the neutrino spectra by, for example, Hyper-Kamiokande experiment and MOON experiment (e.g., Nomachi et al. 2003) will surely give crucial information on the mechanism of core-collapse supernovae. Signature of anisotropy in the neutrino radiation field may be caught in these future facilities together with future gravitational wave detections from supernovae.

\section{References}

Buras, R., Rampp, M., Janka, H.-Th. \& Kifonidis, K. 2003, Phys. Rev. Lett., 90, 241101

Burrows, A., Hayes, J. \& Fryxell, B.A. 1995, Astrophys. J., 450, 830

Colgate, S.A. \& White, R.H. 1966, Astrophys. J., 143, 626

Fryer, C.L. \& Heger, A. 2000, Astrophys. J., 541, 1033

Fryer, C.L. \& Warren, M.S. 2002, Astrophys. J., 574, L65

Fukuda, I. 1982, PASP, 94, 271

Herant, M., Benz, W., Hix, W.R., Fryer, C.L. \& Colgate, S.A. 1994, Astrophys. J., 435, 339

Janka, H.-T. \& Müller, E. 1995, Astrophys. J., 448, L109 
Janka, H.-T. \& Müller, E. 1996, Astron. Astrophys., 306, 167

Kotake, K., Yamada, S. \& Sato, K. 2003, Astrophys. J., 595, 304

Liebendörfer, M., Mezzacappa, A., Thielemann, F.-K., Messer, O.E.B., Hix, W.R. \& Bruenn, S.W. 2001, Phys. Rev. D63, 103004

Madokoro, H., Shimizu, T. \& Motizuki, Y. 2003, Astrophys. J., 592, 1035

Madokoro, H., Shimizu, T. \& Motizuki, Y. 2004, submitted to Publ. Astron. Soc. Japan (astro-ph/0312624)

Mezzacappa, A., Calder, A.C., Bruenn, S.W., Blondin, J.M., Guidry, M.W., Strayer, M.R. \& Umar, A.S. 1998, Astrophys. J., 495, 911

Miller, D., Wilson, J.R. \& Mayle, R. 1993, Astrophys. J., 415, 278

Nomachi, M., Doe, P., Ejiri, H., et al. 2003, in Proc. The 1st Yamada Int. Symp. on Neutrinos and Dark Matter in Nuclear Physics, eds. H. Ejiri and I. Ogawa, http://ndm03.phys.sci.osaka-u.ac.jp/proc/index.htm Session III-12

Shimizu, T., Yamada, S. \& Sato, K. 1993, Publ. Astron. Soc. Japan, 45, L53

Shimizu, T. M., Ebisuzaki, T., Sato, K. \& Yamada, S. 2001, Astrophys. J., 552, 756

Shimizu, T., Yamada, S. \& Sato, K. 1994, Astrophys. J., 432, L119

Wang, L., Wheeler, J.C., Hoeflich, P., et al. 2002, Astrophys. J., 579, 671

Wilson, J.R. \& Mayle, R.W. 1988, Phys. Rep., 163, 63

Wilson, J.R. \& Mayle, R.W. 1993, Phys. Rep., 227, 97 